\section{Hilfreiches Licht beim Speiseröhrenkrebs}

\author{
Plattenepithelkarzinome des Ösophagus werden meist erst in \\ fortgeschrittenen Stadien diagnostiziert. Eine neue optische \\ Technik verbessert die Früherkennung oberflächlich gelegener \\ Tumoren.
}

C ie Suche nach Plattenepithelkarzinomen erfolgt in der Regel endoskopisch mit konventionellem Weißlicht. Als neue Option bietet sich Schmalbandlicht (Narrow Band Imaging, NBI, 414-540 nm) für die Endoskopie an. Die Kombination von Schmalbandlicht und endoskopischer Vergrößerung macht Veränderungen der oberflächlichen mikrovaskulären Struktur besser erkennbar.

320 Patienten mit bestehenden oder früheren Plattenepithelkarzinomen des Ösophagus nahmen an der vorliegenden Studie teil. Randomisiert wurden sie entweder zuerst mit Weißlicht und dann mit Schmalbandlicht oder in umgekehrter Reihenfolge endoskopiert. Neben Ösophagus und gastroösophagealem Übergang wurden auch Oro- und Hy-

Kommentar: In der kurativen Therapie von Kopf-Hals-und Ösophaguskarzinomen kommt der frühzeitigen Diagnostik besondere Bedeutung zu. So werden bis heute Kopf-Hals-Karzinome häufig erst in fortgeschrittenen Stadien erkannt, was für die stagnierenden Überlebenszahlen dieser Tumoren mitverantwortlich ist. In diesem Zusammenhang wird die Bedeutung neuer Techniken deutlich, die eine frühzeitige Diagnose dieser Tumoren ermöglichen.

Schmalbandlicht (Narrow Band Imaging, $\mathrm{NBI}$ ), hat sich in den zurückliegenden Jahren als geeignetes Verfahren zur Erkennung von Frühstadien von Karzinomen in zahlreichen Lokalisationen herauskristallisiert. Hierbei werden breite Spektren von Rot-, Grün- und Blaulicht gefiltert und so frühe Veränderungen der Schleimhaut und die damit einhergehende Neoangiogenese präziser dargestellt. In den letzten zwei Jahren konnte auch für die einzelnen Regionen des Kopf-Hals-Bereichs die diagnostische Überlegenheit des NBI im Vergleich zur konventionellen endoskopischen Weißlichtdiagnostik (White Light Imaging, WLI) nachgewiesen werden. popharynx untersucht. Nur Patienten nach mukosaler Resektion - jedoch nicht nach chirurgischer Resektion oder Chemo-/Radiotherapie - nahmen teil. Bei fraglichen Läsionen wurden Biopsien entnommen und histologisch ausgewertet.

Mit dem Schmalbandlicht als primärer Diagnostik wurden im Ösophagus $97 \%$ der oberflächlichen Karzinome erkannt, mit Weißlicht dagegen nur $55 \%$, im Oround Hypopharynx lagen die entsprechenden Werte bei 100 bzw. $8 \%$. Diese Unterschiede waren mit $\mathrm{p}<0,001$ jeweils statistisch signifikant.

Sensitivität und Spezifität der Schmalbandlicht-Endoskopie werden für die Ösophagusschleimhaut mit 97,2 und $42 \%$, für den Oro- und Hypopharynx mit

Diese Studie, die sowohl bezüglich des Designs als auch statistisch gut konzipiert ist, bestätigt diesen Trend. Der Theorie der Feldkanzerisierung Rechnung tragend werden hier mittels NBI signifikant mehr Zweitmalignome in Frühstadien diagnostiziert als mit konventioneller Weißlichtdiagnostik. Der Unterschied in der Sensitivität ist beeindruckend: Während für WLI Werte von 7,7\% (Kopf-Hals-Bereich) und 55,2\% (Ösophagus) gemessen wurden, liegt die Sensitivität für NBI bei 100 bzw. 97,2\%.

Die Studie untersuchte lediglich neu diagnostizierte Ösophagustumoren bzw. Zweittumoren in Frühstadien. Wenngleich dem Nachweis von Zweitmalignomen bei der Primärdiagnostik ebenfalls große Bedeutung zukommt, so ist v.a. auch der frühzeitige Nachweis möglicher Rezidive nach der Therapie von Kopf-Hals-Malignomen eine große Herausforderung, nicht zuletzt aufgrund veränderter anatomischer Verhältnisse. Gerade hier wäre eine weiterführende wenig aufwendige endoskopische Methode mit präziserer Beurteilung der Schleimhautoberfläche wie mittels Schmalbandlicht von großem Nutzen. Da vorbehandelte Patienten aus der Studie
100 und $79 \%$ angegeben. Die Spezifität der Weißlicht-Endoskopie lag zwar jeweils höher, jedoch ohne statistische Signifikanz.

Fazit: Die Endoskopie mit Verwendung von Schmalbandlicht war in dieser Studie der konventionellen Weißlicht-Endoskopie in der Sensitivität deutlich und signifikant überlegen. Die Autoren sehen in der neuen Untersuchungsmethode einen möglichen neuen Standard.

af

Muto $\mathrm{M}$ et al. Early detection of superficial squamous cell carcinoma in the head and neck region and esophagus by narrow band imaging: A multicenter randomized controlled trial. J Clin Oncol 2010; 28: 1566-72. ausgeschlossen wurden, bleibt eine Aussage hierzu noch unbeantwortet.

Auch der Nutzen des NBI im Rahmen möglicher Screeninguntersuchungen von Hochrisikogruppen (z. B. mit Konsum von Tabakrauch, Alkohol und/oder Exposition gegenüber weiteren Arbeits-und Umweltstoffen) ist nicht zuletzt aufgrund der zurückliegenden Diskussion in anderen Fachbereichen vorerst kritisch zu bewerten.

Insgesamt zeigt die vorliegende Untersuchung, wie auch frühere Studien zu diesem Thema, dass der Einsatz von Schmalbandlicht eine zukunftsweisende Diagnostik zur Früherkennung von Kopf-Hals-Malignomen darstellt, auch wenn der Nutzen für die allgemeine klinische Routine in weiteren Untersuchungen noch geklärt werden muss.

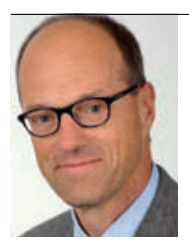

Prof. Dr. med. Heinrich Iro Klinik für Hals-Nasen-Ohrenheilkunde, Universitätsklinik Erlangen Email: heinrich.iro@ukerlangen.de 